\title{
Space Charge Dynamics in Pressboard-oil- pressboard Multilayer System Under DC Voltages
}

\author{
Mingli FU, Bing LUO, Shuai HOU, Yifan LIAO \\ Electric Power Research Institute \\ China Southern Power Grid \\ Guangzhou, CHINA \\ houshuai@csg.cn
}

\author{
Miao HAO and George CHEN \\ School of Electronics and Computer Science \\ University of Southampton \\ Southampton, UK \\ gc@ecs.soton.ac.uk
}

\begin{abstract}
Converter transformers play a critical role in HVDC power transmission system. Space charge is a general concern for dielectric system under DC field. In this paper, the space charge behavior in a sandwiched oil-pressboard insulation system has been investigated using the pulsed electro-acoustic (PEA) method. The presence of homo-charges in the pressboards bulk and hetero-charges at the two interfaces between oil gap and pressboards leads to the electric field enhancement in the pressboards but reduction in the oil gap. The electric field enhancement is much larger than the value estimated by the Maxwell-Wagner polarization.
\end{abstract}

Keywords-HVDC; oil-pressboard; space charge; PEA; interface.

\section{INTRODUCTION}

HVDC converter transformers play a very important role in large scale power transmission systems as well as in renewable energy connections such as off-shore wind-farms. Converter transformers are usually operated under much complex conditions compared with traditional transformers, e.g. the second (or valve) winding should withstand both $\mathrm{AC}$ and DC voltages, and also the polarity reversal voltages [1]. Due to the highly required reliability of converter transformers, the researches on the insulation system of converter transformers have been intensified in the recent years. Oil and pressboard are the common dielectric materials applied in a converter transformer as the main insulation system. In recent years, the space charge behavior in oilpaper/pressboard insulation has been investigated by the pulsed electro-acoustic (PEA) technique [2-7]. Research has shown that the space charge can be formed in the oil/paper insulation system when subject to DC voltage. It is generally believed that the space charge accumulation can distort the local electric field in the dielectrics and lead to acceleration of ageing and even breakdown [8]. The investigation on the space charge dynamics in an oil combined pressboard layers revealed that the interfacial effects between oil and impregnated paper/pressboard might enhance the charge accumulation at the interface and prevent charge dissipation $[9,10]$. However, oil/paper/pressboard combination insulation is much more complex in a real transformer, i.e. the oil gap presents within multilayers of pressboards, leading to more interfaces between oil and pressboard. The space charge behavior in this complex insulation structure is still poorly understood.

In this paper, a sandwiched insulation system, consisted of two layers of $0.5 \mathrm{~mm}$ impregnated pressboards and one layer of $0.5 \mathrm{~mm}$ oil gap, is used to present the multilayer insulation in a real converter transformer, and the space charge characteristics are investigated by a purpose built PEA system under room temperature.

\section{EXPERMENTAL SETUP}

\section{A. Sample preparation}

In order to investigate the impacts of oil status on space charge dynamics, fresh mineral oil and service aged oil were used in this work. The fresh oil has high oxidation stability and low sulphur content to meet IEC 60296. The aged oil was collected from a long term serviced power transformer. The major properties of the two types of oil are shown in Table I.

TABLE I. STATUS OF THE OILS

\begin{tabular}{|c|c|c|}
\hline Parameters & Fresh Oil & Aged Oil \\
\hline Moisture (ppm) & 3.8 & 4.3 \\
\hline 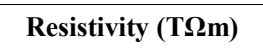 & 4 & 0.1 \\
\hline Relative Permittivity & 2.2 & 2.6 \\
\hline Colour & Transparent & Dark brown \\
\hline
\end{tabular}

The pressboards used in this work were the $0.5 \mathrm{~mm}$ thick general available transformer pressboards with smooth surface. The pressboards were cut into circular with a diameter of $9 \mathrm{~cm}$, and dried at $353 \mathrm{~K}$ for 3 days. Then, the dried pressboards were separately impregnated in the two types of oil under $10 \mathrm{~Pa} / 333 \mathrm{~K}$ for another 3 days to ensure the pressboards were fully impregnated with oil. The used oil and impregnated pressboards were kept in a seal container under vacuum condition to avoid absorbing moisture and impurities. The major dielectric properties of the impregnated pressboards are shown in Table II.

TABLE II. DIELECTRIC PARAMETERS FOR IMPREGNATED PRESSBOARDS

\begin{tabular}{|c|c|c|}
\hline Parameters & Fresh Oil & Aged Oil \\
\hline Relative Permittivity & 3.2 & 4.2 \\
\hline 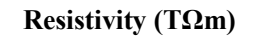 & 100 & 0.3 \\
\hline
\end{tabular}




\section{B. Experimental method}

The PEA system used in this work was purposed built for overcoming the difficulty of large acoustic attenuation due to the thick sample and the formation of oil gap between two layers of pressboards. The piezoelectric sensor used in this system was a PVDF film with a thickness of $40 \mu \mathrm{m}$ and an area of $0.8 \mathrm{~cm}^{2}$. The ground electrode was made of aluminum plate with a thickness of $1 \mathrm{~cm}$, and it was modified to a container with a depth of $0.5 \mathrm{~cm}$ and a diameter of $10 \mathrm{~cm}$ that allows to contain both solid and liquid dielectric materials. As shown in Fig. 1, the oil gap was formed by a PTFE ring with a thickness of $0.5 \mathrm{~mm}$, which is sandwiched between two layers of impregnated pressboard. The pulse voltage used in this work was $1 \mathrm{kV}, 2 \mathrm{kHz}$ with $5 \mathrm{~ns}$ width. Compared with the applied DC testing voltage, the possible charge accumulation caused by the pulse voltage can be ignored.

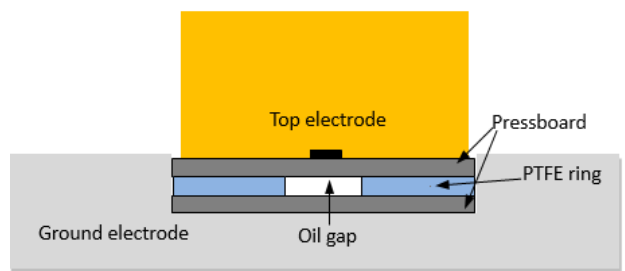

Fig. 1. The struture of insulation system and electrode system

The DC electric fields applied in the experiments were 20 $\mathrm{kV} / \mathrm{mm}$ for fresh oil samples and $16 \mathrm{kV} / \mathrm{mm}$ for aged oil samples. Flashover occurred in the aged oil samples at the applied electric field of $16.7 \mathrm{kV} / \mathrm{mm}$.

\section{SPACE CHARGE RESULTS}

\section{A. Volt-on results}

The volt-on measurements were carried out during the DC electric field application, which recorded the charge build-up process under the external electric field.

Fig. 2 shows the space charge dynamics in fresh oil sample under $20 \mathrm{kV} / \mathrm{mm}$. In the first layer of pressboard that contacts with the ground electrode, homo charge injection occurs, i.e. negative charges inject from electrode into the pressboard bulk. The cathode peak drops with the external voltage application from $12 \mathrm{C} / \mathrm{m}^{3}$ to $9.8 \mathrm{C} / \mathrm{m}^{3}$. The injected negative charges move from cathode side to the oil gap side, and broadly distributed throughout the thickness of the pressboard within 1 hour. On the other hand, in the second layer of pressboard that directly contacts with the anode(top electrode), the positive peak moves towards to the oil gap indicating positive charges injected from the anode into the pressboard bulk. However, the injected charges are difficult distinguished from the electrode peak due to large acoustic attenuation and dissipation at the anode side.

Considerable charges gradually accumulate at the interfaces between oil gap and pressboards with the voltage application. Positive charges accumulate at the first interface between the oil gap and the first layer of pressboard, while negative charges accumulate at the other interface between the oil gap and the second layer of pressboard. The amplitudes of the both interfacial peaks keep increasing during the 1 hour volt-on test, which enhances the electric field distributed in the regions within the two layers of pressboards near the interfaces, but decreases the electric field in the oil gap. This agrees with the Maxwell-Wagner polarization that charges will be formed at the interface when the dielectrics have discontinuity of the ratio of permittivity and conductivity.

As shown in Fig. 3, the space charge dynamics characteristics in the aged oil sample under $16 \mathrm{kV} / \mathrm{mm}$ is significant enhanced. It can be observed that homo charge injection greatly occurs in the first 5 minute, e.g. the cathode peak drops from about $9.2 \mathrm{C} / \mathrm{m}^{3}$ to $6.2 \mathrm{C} / \mathrm{m}^{3}$ in the first 5 minute, and then to about $4.3 \mathrm{C} / \mathrm{m}^{3}$ at the 60 minute. Both of the cathode peak and anode peak move into the bulk of the pressboards.

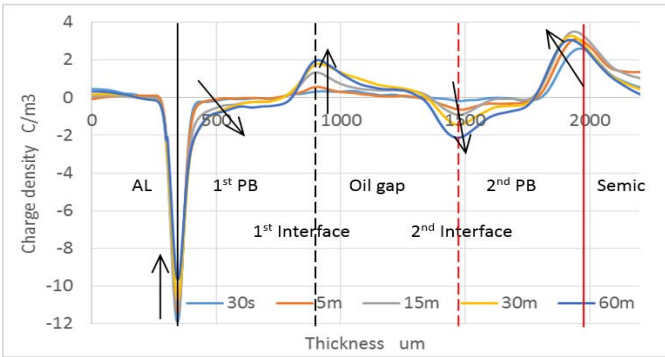

Fig. 2. Volt-on results in the fresh oil sample under $20 \mathrm{kV} / \mathrm{mm}$

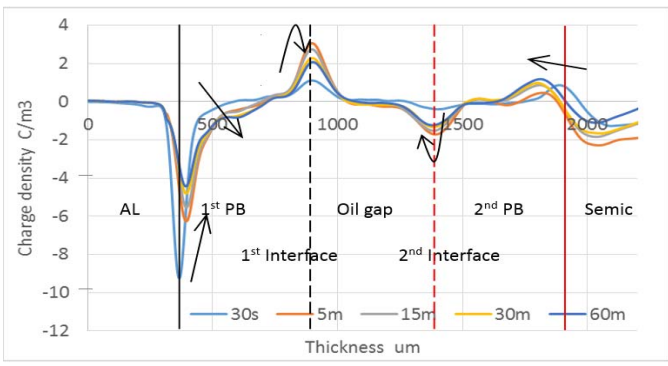

Fig. 3. Volt-on results in the agde oil sample under $16 \mathrm{kV} / \mathrm{mm}$

Similar with the fresh oil sample, positive charges accumulate at the first interface and negative charge accumulate at the second interface. However, the amplitudes of the two interfacial peaks in aged sample quickly increase within the first 5 minute and then gradually decrease until the saturation state is reached. For example, the amplitude of the first interfacial peak dramatically raises from about $1 \mathrm{C} / \mathrm{m}^{3}$ to $3 \mathrm{C} / \mathrm{m}^{3}$ within the first 5 minute and then drops to about 2 $\mathrm{C} / \mathrm{m}^{3}$ during the 1 hour voltage application.

\section{B. Charge decay results}

After the 1 hour voltage application, the decay tests were carried out by removing the external voltage application, and the results reveal the real space charge profile throughout the insulation. It is usually believed that the space charge dynamics without the influence of the external voltage can reflect the trap energy levels of dielectric materials.

The space charge decay characteristics in fresh oil sample are shown in Fig. 4. It can be observed that negative charges distribute in the bulk of the first layer pressboard, while some positive charges accumulate in the second layer pressboard near the anode. This confirms the homo charge injection when the external voltage is applied. It is also found that positive 
charges presence at the first interface between oil gap and pressboard, while negative charges locate at the second interface. With the 1 hour depolarization time, the accumulated charges only slightly decrease, suggesting the mobility of the accumulated charges in fresh oil is generally small.

On the other hand, Fig. 5 shows the charge dissipation dynamics in aged oil sample. At the initial time, compared with fresh oil, more space charges accumulated in the multilayer insulation system, e.g. in the first layer of pressboard, the amplitude of the negative peak is about $3.9 \mathrm{C} / \mathrm{m}^{3}$ and the negative charges widely distribute in the whole bulk of the pressboard, but there was a good similarity in space charge profiles between the fresh and aged oil samples. Similarly, significant positive charge distribution can be observed in the second layer of pressboard. Electric field near the two interfaces of the oil gap is greatly enhanced due to the opposite polarities charges distribute near the interfaces in the oil gap and pressboard. With the decay time, the accumulated charges dissipate very quickly, and leave only background noise after 30 minutes, indicating the mobility of accumulated charges is much greater than that of the fresh oil sample.

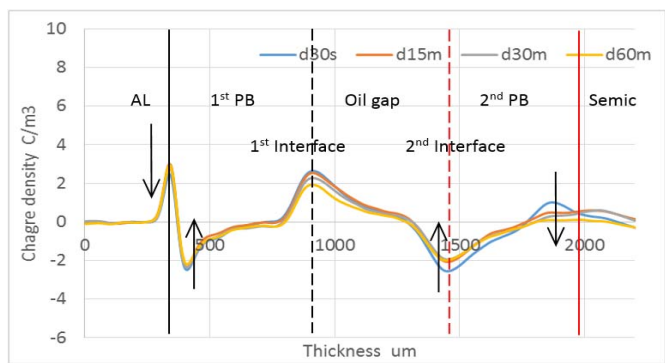

Fig. 4. Decay results in the fresh oil sample

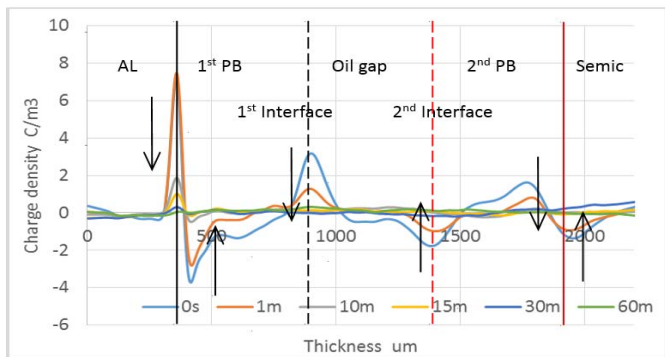

Fig. 5. Decay results in the aged oil sample

\section{ANALYSIS AND DISSICATION}

\section{A. Total absolute charge amount}

The absolute charge quantity show the space charge accumulation or dissipation in the sample with the time, which can be used to compare the charge characteristics in different samples and materials properties. The charge amount can be easily calculated from the measured space charge profiles based on the equation:

$$
Q(t)=\int_{0}^{d}|\rho(x, t)| S d x
$$

where $d$ is the thickness of the sample, $\rho(x, t)$ is the charge density, $S$ is the area of the sample covered underneath the electrode.
Based on above equation, the results of absolute charge amount vs. time for volt-on and decay processes are shown in Fig. 6 and Fig. 7.

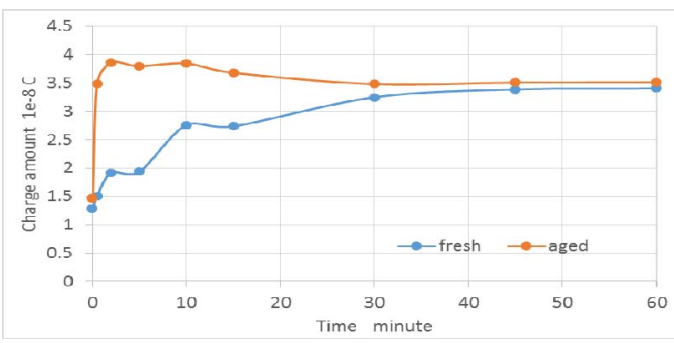

Fig. 6. Total charge amount vs. volatge application time

As shown in Fig. 7, the bottom line indicates the total charge amount in the fresh oil sample under $20 \mathrm{kV} / \mathrm{mm}$, while the top line is the charge amount in aged oil sample under 16 $\mathrm{kV} / \mathrm{mm}$. The charge amount in fresh oil sample keeps gradually increasing almost over the whole period of voltage application time, and becomes stable after 40 minute. On contrary, the charge amount in the aged oil sample dramatically jumps to the maximum value (about $3.9 \times 10^{-8} \mathrm{C}$ ) in the first 2 minute, then slowly decreases to a stable value (about $3.5 \times 10^{-8} \mathrm{C}$ )

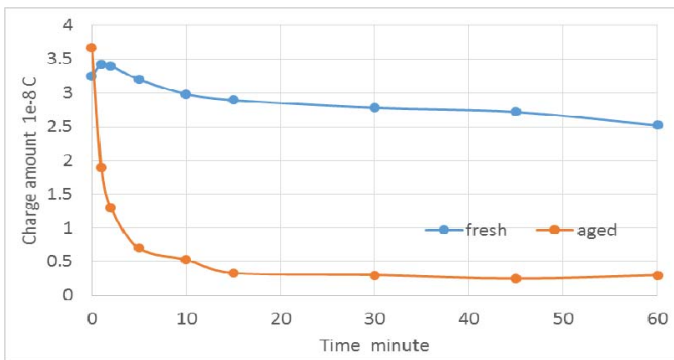

Fig. 7. Total charge amount vs. charge dissipation time

The results of charge quantity reduction during the decay process are shown in Fig. 7. The charge dissipation in the fresh oil sample is very slow, i.e. the charge amount is decreased from about $3.5 \times 10^{-8} \mathrm{C}$ to $2.5 \times 10^{-8} \mathrm{C}$ over 1 hour of time. Whereas the charge decay in aged oil sample is much faster, and most of the charge died off within the first 5 minutes with the total charge dropping from about $3.6 \times 10^{-8} \mathrm{C}$ to $0.6 \times 10^{-8} \mathrm{C}$, and then gradually reduced to $0.3 \times 10^{-8} \mathrm{C}$ almost the measurement noise in the rest 55 minutes.

\section{B. Electric field distribution and distortion}

The presence of space charge can distort the electric field distribution along the samples, therefore, it is important to understand the influence of space charge to electric field profile within a specific insulation system.

The electric field distribution can be calculated by the integration of the charge density throughout the insulation thickness and is expressed as below:

$$
E(x)=\int_{0}^{x} \frac{\rho(x)}{\varepsilon_{0} \varepsilon_{r}} \mathrm{~d} x \quad 0 \leq x \leq d
$$

where $\rho(x)$ is the charge density, $\varepsilon_{0}$ is the vacuum permittivity, $\varepsilon_{\mathrm{r}}$ is the relative permittivity of test sample, $d$ is the thickness of the sample. 
Generally, the accumulation of positive charges at the first interface, while negative charges at the second interface, leading to the electric field enhancement in the two layers of pressboard, but the field reduction in the oil gap. Moreover, the homo charge injection from the both electrodes can reduce the electric field distributes in the region that between electrodes and pressboards. However, the opposite polarity charges accumulated near the interfaces between oil gap and pressboards can greatly enhance the electric field near the interfaces. Particularly, this may be the case in the aged oil samples, i.e. the large amount homo charges can be quickly injected and move through the pressboard bulk and meet the interfacial peak at the interfaces due to the high conduction of aged oil. This results in a considerable field enhancement at the interfaces might be the reason for the flashover taken place in aged oil at the electric field level of $16.7 \mathrm{kV} / \mathrm{mm}$.

The distortion factor of the electric field $\Delta E$ can be calculated by the equation:

$$
\Delta E=\frac{E_{\max }-E_{a v}}{E_{a v}} \times 100 \%
$$

where the $E_{\max }$ is the maximum value of the electric stress in the dielectric bulk due to the space charge accumulation; $E_{\text {av }}$ is the average value of the applied electric stress.

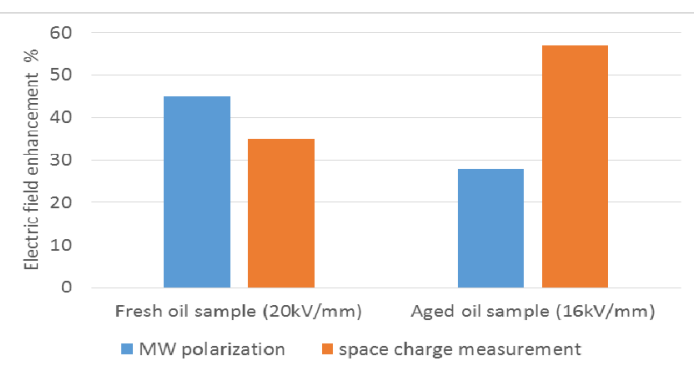

Fig. 8. Electric field enhancements in different samples.

Figure 8 shows the comparison of electric field enhancements between the space charge measured results and the estimation based on the Maxwell-Wagner (MW) polarization. In the MW polarization, the electric field distributions in different layers of dielectrics are reverse proportional with the conductivities of each layer material, when the steady state is reached. According to Figure 8 , for the fresh oil sample, the field distortion based on the space charge profiles is smaller than the Maxwell theory. The space charge dynamics is highly related with the time of the voltage application. Therefore, in this work, the 1 hour voltage application time may be not sufficient for the charge accumulation, leading to a small electric field distortion.

On the other hand, the electric field enhancement in the aged oil sample shows the great different between the two methods. It indicates that the severe electric field distortion is related with the dielectric properties, rather than the ratio of the conductivity.

\section{Conclusions}

In this present work, the space charge behavior in multilayer sandwiched pressboard-oil-pressboard insulation system is investigated. Homo charge injection has been observed for all the samples, i.e. negative charges distribute in the first layer of pressboard and positive charges accumulate in the second layer of pressboard. Positive interfacial peak occurs at the interface between the oil gap and first layer of pressboard, while negative one occurs at the second interface, which increases the electric field in the pressboard but decreases the field in the oil gap. The space charge accumulation in the aged oil sample is much stronger and quicker than in the fresh oil sample, while the charge dissipation is also quicker. Therefore, the electric field distortion in the aged oil sample is larger than in the fresh oil sample though the applied electric field is lower. Compared with the field enhancements estimated by Maxwell-Wagner polarization, the enhancements calculated based on the measured space charge profiles are highly related with the dielectric properties themselves, rather than the ratio of the conductivities, especially in the aged oil sample, suggesting an unexpected risk on the insulation system.

\section{ACKNOWLEDGMENT}

MH, GC would like to thank the financial support from China Southern Power Grid Co. Ltd (NEL201402).

\section{REFERENCES}

[1] C. Kim, V. Sood, G. Jang, S. Lim and S. Lee, HVDC Transmission: Power Conversion Applications in Power Systems, Chichester: John Wiley \& Sons, Ltd, 2009.

[2] M. Jeroense and P. Morshuis, "Space Charge Measurements on Impregnated Paper: A Review of the PEA Method and a Discussion of Results," IEEE Electrical Insulation Magazine, vol. 13(3), pp. 26-35, May 1997.

[3] R. Ciobanu, I. Prisecaru and S. Aradoaei, "PEA Measurements upon Cellulose Materials Submitted to Gamma Radiation," in International Conference on Solid Dielectrics, France, 2004.

[4] C. Tang, G. Chen, M. Fu and R. Liao, "Space Charge Behavior in Multilayer Oil-paper Insulation under Different DC Voltages and Temperatures," IEEE Transactions on Dielectrics and Electrical Insulation, vol. 17(3), pp. 778-788, 2010.

[5] J. Hao, G. Chen, R. Liao, L. Yang and C. Tang, "Influence of Moisture on Space Charge Dynamics in Multilayer Oil-paper Insulation," Dielectrics and Electrical Insulation, vol. 19(4), pp. 1456 - 1464, Aug 2012.

[6] Y. Zhou, Y. Wang, G. Li, N. Wang, Y. Liu, B. Li, P. Li and H. Cheng, "Space Charge Phenomena in Oil-paper Insulation Materials under High Voltage Direct Current," Journal of Electrostatics, vol. 67, pp. 417-421, 2009.

[7] M. Hao, Y. Zhou, G. Chen, G. Wilson and P. Jarman, , "Space charge behavior in thick oil-impregnated pressboard under HVDC stresses," IEEE Trans. Dielectr. Electr. Insul., vol. 22, no. 1, pp. 72-80, 2015.

[8] X. Wu, G. Chen, A. E. Davies, Y. Tanaka, S. J. Sutton and S. G. Swingier, "Space charge measurements in polyethylene under DC and AC operating conditions using the PEA technique," Dielectric Materials, Measurements and Applications, pp. 57-62, 2000.

[9] K. Wu, Q. Zhu, H. Wang, X. Wang and S. Li, "Space charge behavior in the sample with two layers of oil-immersed-paper and oil," IEEE Trans. Dielectr. Electr. Insul., vol. 21, no. 4, pp. 1857-1865, 2014.

[10] M. Hao; Y. Zhou; G. Chen; G. Wilson and P. Jarman, "Space charge behaviour in oil and impregnated pressboard combined insulation system", Dielectric Liquids (ICDL) IEEE International Conference on, 2014 . 